Journal of Theoretical and Applied Mechanics, Sofia, 2015, vol. 45, No. 1, pp. 17-38

\title{
ELECTRIC VEHICLES MILEAGE EXTENDER KINETIC ENERGY STORAGE*
}

\author{
Venelin Jivkov, Vutko Draganov, Yana Stoyanova \\ Department of Theory of Mechanisms and Machines, Technical University of Sofia, \\ 8, Kl. Ohridski Blvd., 1797 Sofia, Bulgaria, \\ e-mails: jivkov@tu-sofia.bg, yast@tu-sofia.bg
}

[Received 31 July 2014. Accepted 09 March 2015]

\begin{abstract}
The proposed paper considers small urban vehicles with electric hybrid propulsion systems. Energy demands are examined on the basis of European drive cycle (NEUDC) and on an energy recuperation coefficient and are formulated for description of cycle energy transfers. Numerical simulation results show real possibilities for increasing in achievable vehicle mileage at the same energy levels of a main energy source - the electric battery. Kinetic energy storage (KES), as proposed to be used as an energy buffer and different structural schemes of the hybrid propulsion system are commented. Minimum energy levels for primary (the electric battery) and secondary (KES) sources are evaluated. A strategy for reduced power flows control is examined, and its impact on achievable vehicle mileage is investigated. Results show an additional increase in simulated mileage at the same initial energy levels.

KEY wORDS: Electric vehicles, hybrid propulsion systems, electric machine - kinetic storage device.
\end{abstract}

\section{Introduction}

Ecology problems, energy crisis and the tendency for decreasing the world existence of fossil fuels, guide the investigators' and manufacturers' efforts to more efficient usage of the energy sources. The different hybrid propulsion systems intended for vehicle applications including the electric vehicles (Mitsubishi, Hyundai, GM, Peugeout, etc.), which are already available on the market, are an illustration of the above statement [1, 2].

\footnotetext{
${ }^{*}$ Corresponding author e-mail: yast@tu-sofia.bg

The authors acknowledge the financial support of Ministry of Education, Youth and Science, contract No DUNK01/3-2009.
} 
Possibilities for energy recuperation during vehicles brake modes are analyzed in the presented paper, and the main attention is paid to vehicles with hybrid propulsion system, where kinetic energy storage (KES) and its appropriate power branch are added to the conventional electric drive line [3].

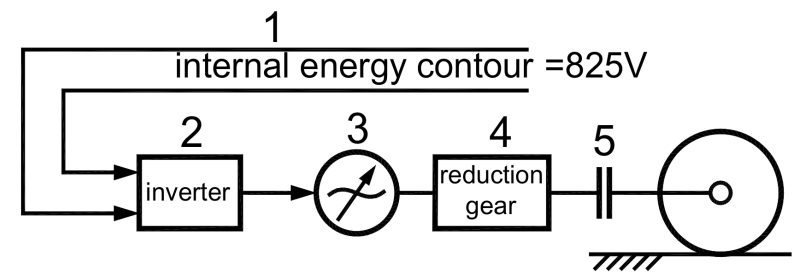

Fig. 1. Typical energy recovery system used in electric railway trains including commuting rails consists of : 1 - internal energy contour; 2 - inverter; 3 - AC motor/generator with frequency control; 4 - reduction gear; 5 - clutch

Necessity to transfer large energy flows for short periods of time (2 to 6 sec for light vehicles and up to $15 \mathrm{sec}$ for trains) is the main challenge with the energy systems intended to store the vehicles energy during their brake modes. Such high stressed loads are beyond the characteristics of the conventional electric batteries. This fact limits the practical application of such systems and they are usually used in electric railway trains, basically commuting rails and metropolitan trains, where the energy during braking is transformed into electrical one, which is sent subsequently to an internal energy circuit (Fig. 1). A similar solution is used in some of the trains in the Metropolitan of Sofia, Bulgaria, for example, where the induction drive system with frequency control is in a generator mode of operation during movement with negative accelerations until specific minimum train velocity is reached, and all the energy is transferred trough the inverter with DC current and $825 \mathrm{~V}$ voltage into the Metropolitan internal energy circuit. Such an approach is not suitable for light vehicles.

Absorbing large power flows with high density is possible only for capacitive (supercapacitors) and kinetic energy storages (superflywheels). Basic disadvantages of the supercapacitors are large dimension and mass parameters, but for the superflywheels - high aerodynamic and mechanical losses over time Problems connected to the superflywheels are successfully solved nowadays by usage of vacuum environment, magnet suspension, etc. [4].

Capacity determination of an energy storage device intended for vehicle application and its efficiency depend entirely on the vehicle operation modes. Different operation modelling modes are based on the so-called drive cycles, 
which define local traffic conditions [5], or ideal but close to real conditions models of vehicle behaviour [6]. Using the drive cycles for comparative analysis, quantity of vehicle emissions for example, enforces some partial standardisation among the cycles, according to different types of environment (urban, extra urban and highway driving conditions), different type of vehicles (light-duty and heavy duty vehicles), and different regions as well (Europe, USA, Japan). The analysis presented here is based on the New European Urban Drive Cycle (NEUDC), which is composed of four ECE-15 cycles simulating city driving; each one cycle has duration of 200 sec., and one Extra Urban Driving Cycle with duration of $400 \mathrm{sec}$, which describes typical highway conditions (Fig. 5).

Many publications explore strategies for usage of the alternative energy sources in the hybrid vehicles [7, 8, 9, 10]. A review article [11] shows this tendency of increased interest in such a topic - more than 50 paper published per year, since 2010. But all of them consider typical hybrid system where an internal combustion engine is used as a primary energy source.

The purpose of the investigation presented here is a determination of KES capacity and its impact on the achievable mileage of a pure electric vehicle over a chosen drive cycle. Analysis is based on energy/power flows modelling and different strategies for KES usage are estimated.

2. Estimation of possibilities for energy recovery and a criterion for direction of the energy flow

The vehicle energy needed to cover a specific drive cycle is defined by:

$$
E_{\Sigma}=\int_{0}^{t c} P_{t} d t+\int_{0}^{t c} P_{f} d t+\int_{0}^{t c} P_{w} d t
$$

where: $P_{t}=m \delta \dot{v} v$ is a power for active change of the vehicle energy state, [W]; $P_{f}=f_{r} m g v$ is a power for overcoming the tire resistance, $[\mathrm{W}] ; P_{w}=$ $0.5 \rho A_{f} C_{d} v^{3}$ is a power for overcoming the aerodynamic resistance, [W], $v$ is a current vehicle speed determined by the drive cycle, $[\mathrm{m} / \mathrm{s}] ; m$ is a vehicle mass, $[\mathrm{kg}] ; g$ is the acceleration of gravity, $9.81\left[\mathrm{~m} / \mathrm{s}^{2}\right] ; \rho$ is the ambient air density, $1,202\left[\mathrm{~kg} / \mathrm{m}^{3}\right] ; C_{d}$ is the vehicle drag coefficient, $[-] ; A_{f}$ is the vehicle frontal area, $\left[\mathrm{m}^{2}\right] ; \delta$ is a coefficient which gives an account on the rotating masses in the vehicle drive line, $[-] ; f_{r}$ is a coefficient of road resistance [-] - [12].

It is possible to receive quantity estimation with considerable accuracy of how much energy could be recovered by comparing the necessity energy flows for vehicle acceleration and deceleration over an ideal drive cycle. The last one 


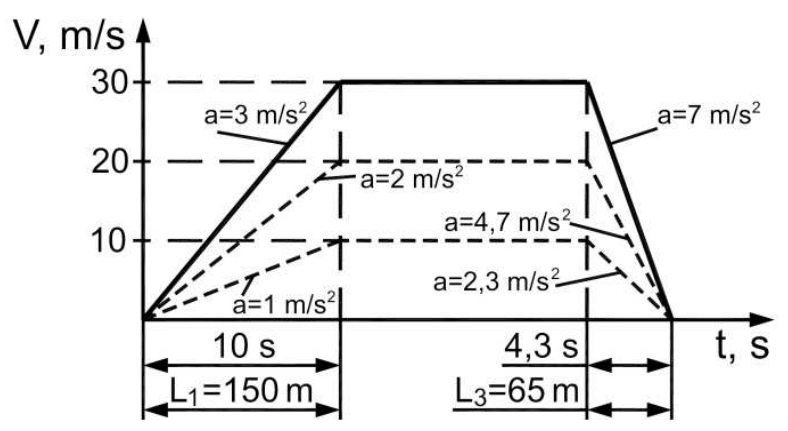

Fig. 2 A simple trapezium drive cycle of the vehicle speed profile

is considered as a combination of different simple trapezium drive cycles which describe the speed profiles versus time, as shown in Fig. 2.

In case of a discrete speed profile, the representation of the energy required to accelerate a unit of the vehicle mass, could be described as:

$$
E_{\dot{v} \geq 0}=\frac{1}{\eta_{1}} \sum_{i=1}^{n-1}\left\{\frac{\delta}{2}\left|v_{i}^{2}-v_{i+1}^{2}\right|+g(f \pm \sin \alpha) \int_{t_{i}}^{t_{i+1}} v d t+k_{v} \int_{t_{i}}^{t_{i+1}} v^{3} d t\right\} .
$$

Similarly, the energy required to be absorbed during braking, could be presented as:

$$
E_{\dot{v}<0}=\eta_{2} \sum_{i=1}^{n-1}\left\{\frac{\delta}{2}\left|v_{i}^{*^{2}}-v_{i+1}^{*^{2}}\right|-g(f \pm \sin \alpha) \int_{t_{i}^{*}}^{t_{i+1}^{*}} v d t-k_{v} \int_{t_{i}^{*}}^{t_{i+1}^{*}} v^{3} d t\right\}
$$

where: the time intervals $t_{i}, t_{i+1}[\mathrm{~s}]$ correspond to the acceleration modes, when the vehicle acceleration $\dot{v}$ is positive or zero; the time intervals $t_{i}^{*}, t_{i+1}^{*}$ $[\mathrm{s}]$ correspond to the braking modes with negative acceleration; $\eta_{1}, \eta_{2}$ are the drive line efficiencies to and from the driven wheels. The air drag coefficient is used in a new form as $k_{v}=0.5 \rho A_{f} C_{d} / m,\left[\mathrm{~m}^{-1}\right]$, which represents the air resistance assigned to an unit of the vehicle mass.

From the ratio between the expressions (3) and (2), it can be judged how much of the exhausted energy for acceleration would be possible to recover in some kind of stored energy. This ratio is considered as a recuperation 
coefficient $k_{r}$, which is presented in the following form:

(4) $k_{r}=\frac{E_{\dot{v}<0}}{E_{\dot{v}>0}}=\eta_{\Sigma} \frac{\sum_{i=1}^{n-1}\left\{\frac{\delta}{2}\left|v_{i}^{*^{2}}-v_{i+1}^{*^{2}}\right|-g(f \pm \sin \alpha) \int_{t_{i}^{*}}^{t_{i+1}^{*}} v d t-k_{v} \int_{t_{i}^{*}}^{t_{i+1}^{*}} v^{3} d t\right\}}{\sum_{i=1}^{n-1}\left\{\frac{\delta}{2}\left|v_{i}^{2}-v_{i+1}^{2}\right|+g(f \pm \sin \alpha) \int_{t_{i}}^{t_{i+1}} v d t+k_{v} \int_{t_{i}}^{t_{i+1}} v^{3} d t\right\}}$.

The recuperation coefficient $k_{r}$ is depicted in Fig. 3 as a function of maximum achievable speed for the cycle in Fig. 2, at $\alpha=0$ (zero road gradient angle), $\eta_{\Sigma}=0.72, k_{v}=4.10^{-4}[1 / \mathrm{m}], \delta=1.1$ and different values of the road resistance coefficient $f$. The same coefficient $k_{r}$ is presented in Fig. 4, but now the gradient angle is considered as a variable for a constant value of $f=0.01$.

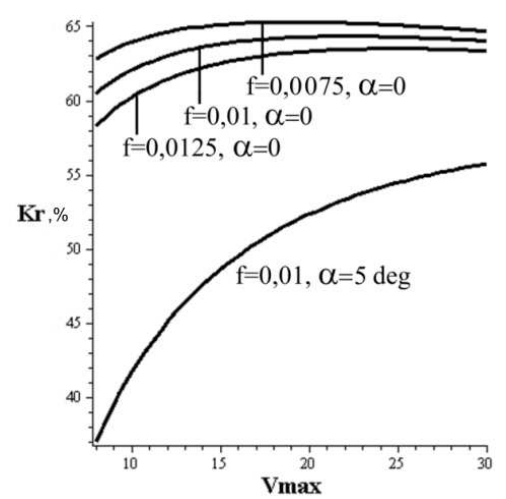

Fig. 3 Recuperation coefficient $k_{r}$ as a function of the cycle maximum speed according Fig. 2 at $\alpha=0$

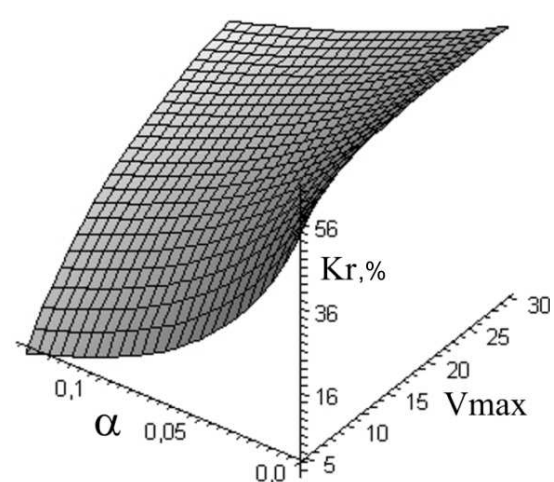

Fig. 4 Recuperation coefficient $k_{r}$ as a function of both the cycle maximum speed according to Fig. 2 and the gradient angle $\alpha$

The results represent the optimum scenario, when there is no vehicle movement with constant speed, and the simplified drive cycle contains only acceleration and deceleration modes. The presented results analysis shows, that for this ideal case, there is a possibility to recover 40 to 60 percent of the energy used for acceleration during brake modes, instead of transforming it into heat at brake system.

In spite of the used drive cycle, the vehicle movement can be described as a combination of three basic driving modes determined by the necessity direction of energy flow [12]. The condition, which allows those modes to be strictly determined, could be obtained from the differential equation of the 
vehicle motion in case of free movement on horizontal road and without any disturbances, but only rolling and air drag resistance forces:

$$
d v / d t=-\alpha^{2} v^{2}-\beta^{2},
$$

at $\alpha=\sqrt{k_{v} / \delta}$ and $\beta=\sqrt{f_{r} g / \delta}$. For $v \geq 0$, i. e. just forward vehicle movement, the equation (5) can be integrated in closed form yielding the result:

$$
v_{u}(t)=\frac{\beta}{\alpha} \tan \left\{\arctan \left[\frac{\alpha}{\beta} v\left(t_{0}\right)\right]-(\alpha \beta) t\right\} .
$$

Transforming the above solution into the discrete form for each time interval from the drive cycle leads to the following expression:

$$
v_{u i}=\sqrt{f_{r} g / k_{w}} \tan \left\{\arctan \left[\sqrt{k_{w} / f_{r} g} v_{i-1}\right]-\frac{\sqrt{k_{w} f_{r} g}}{\delta} \Delta t_{i}\right\} .
$$

The results from (6) can be used to define the operational mode of the vehicle, during each discrete time interval from the cycle and the direction of energy flow, respectively (Fig. 5). The different modes are determined by quantity comparison between defined speed at the end of every time interval $i-1, i$ from the drive cycle and estimated speed at the end of the same interval according to expression (6):

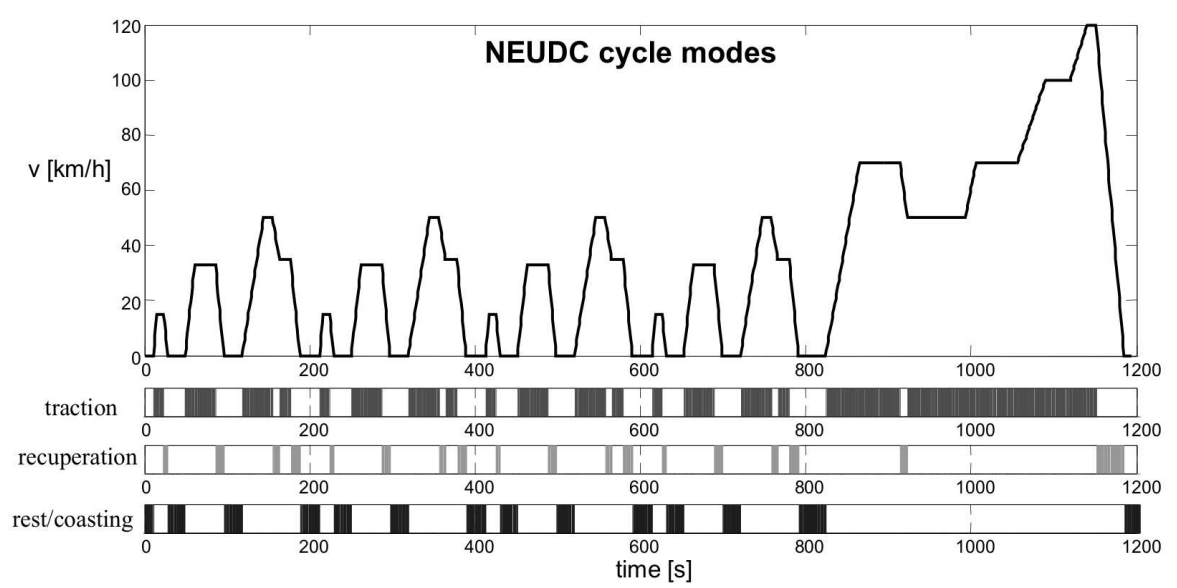

Fig. 5 Speed profile of the NEUDC drive cycle and corresponding operation modes

$v_{i}>v_{u i}$ - necessity for energy supply for fulfilment the defined motion (traction mode); 
On the Modulation of Oscilation in Thermohaline Convection Problems ...23

$v_{i}<v_{u i}$ - necessity for energy deprivation for fulfilment the defined motion (braking mode);

$v_{i}=v_{u i}$ - without energy transfer (coasting mode).

The described recuperation coefficient (4) and expressions (2) and (3) are suitable for a single drive line with just one energy source included, because of taking into consideration the overall drive line efficiency. For parallel drive lines with more than one energy source, the recuperation coefficient have to be reworked. For that purpose, the recuperation coefficient $k_{r}$ is transformed into a coefficient of energy recovery at the drive wheels, taking into account again the direction of the energy flows:

$$
k_{E}=\frac{\left|\Sigma E_{v i<v u i}\right|}{\Sigma E_{v i>v u i}},
$$

where: $\Sigma E_{v i<v u i}$ is the overall energy available for recuperation, but $\Sigma E_{v i \geq v u i}$ is the energy required to fulfil movement over the determined drive cycle. Comparing the expressions (4) and (7) leads to the following relation in case of a conventional electric drive line:

$$
k_{r}=\eta_{\Sigma} \frac{\left|\Sigma E_{\dot{v}<0}\right|}{\Sigma E_{\dot{v}>0}} \approx \eta_{\Sigma} \frac{\left|\Sigma E_{v i<v u i}\right|}{\Sigma E_{v i>v u i}}=\eta_{\Sigma} k_{E} .
$$

The coefficient $k_{E}$, expression (7), is a functional characteristic of the drive cycle and of the vehicle resistances coefficients. As $k_{E}$ is considered as an energy transfer coefficient assigned to the vehicle drive wheels, this coefficient does not depend on the efficiency of the energy transfers among energy sources and the vehicle wheels.

Typical values of $k_{E}$ for the European NEUDC drive cycle are presented in Table 1, which describe six different types of vehicles suitable for usage of electric propulsion system [12]: 1 - sport utility vehicle; 2 - full size sedan; 3 - middle size sedan; 4 - compact vehicle; 5 - small multi-purpose vehicle; 6 - a small bus. Data for $k_{E}$ are related to the end of the NEUDC cycle. It

Table 1. Values of $k_{E}$ for different types of vehicles

\begin{tabular}{|l|l|l|l|l|l|l|}
\hline & 1 & 2 & 3 & 4 & 5 & 6 \\
\hline$A_{f} C_{d}\left[\mathrm{~m}^{2}\right]$ & 1.2 & 0.7 & 0.6 & 0.4 & 0.7 & 1.88 \\
\hline$f_{r}[-]$ & 0.017 & 0.013 & 0.012 & 0.008 & 0.013 & 0.015 \\
\hline$m[\mathrm{~kg}]$ & 2000 & 1500 & 1000 & 750 & 1700 & 4436 \\
\hline$k_{v}\left[\mathrm{~m}^{-1}\right] 10^{-4}$ & 3.6 & 2.8 & 3.6 & 3.2 & 2.47 & 2.54 \\
\hline$k_{E}[-]$ & 0.2131 & 0.2805 & 0.2608 & 0.3277 & 0.3073 & 0.2693 \\
\hline
\end{tabular}




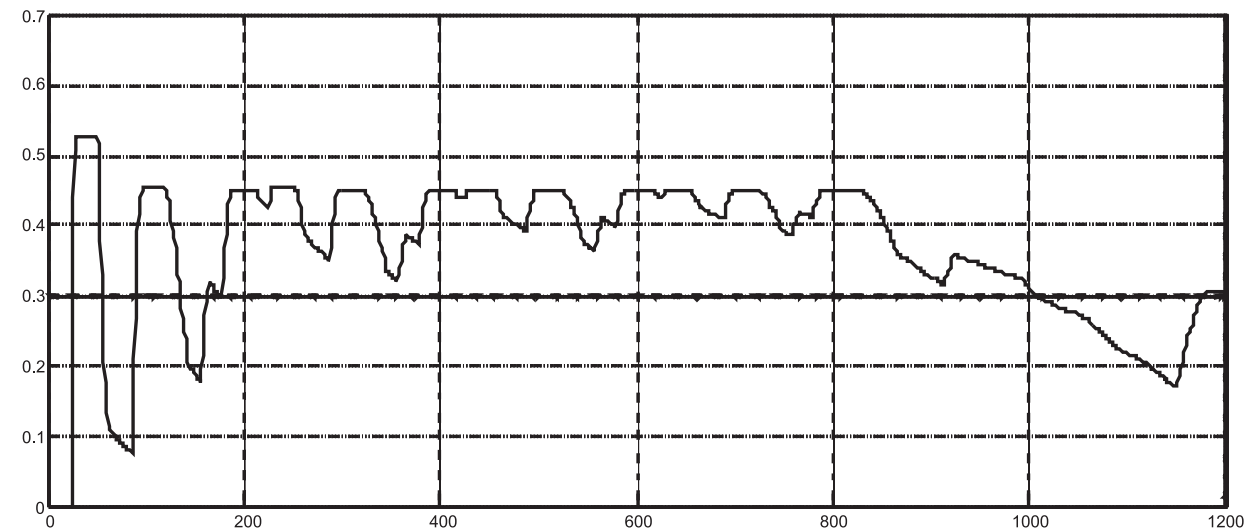

a) $n=1$
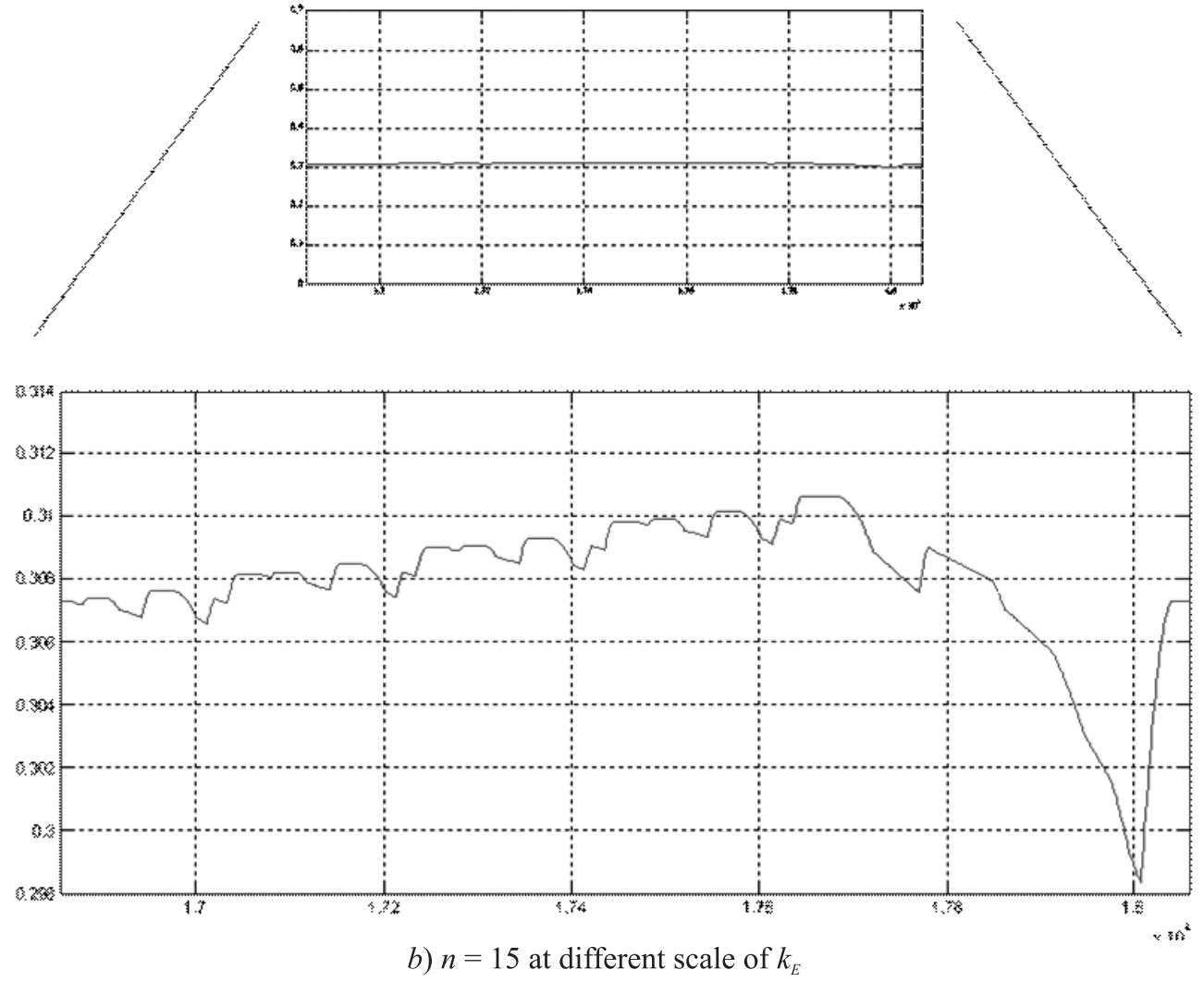

Fig. 6. Evolution of $k_{E}$ during motion over reiterated NEUDC cycles 
is assumed that any predetermined mileage is accomplished by repeating the same drive cycle. This repeating decreases the variation of the coefficient $k_{E}$ and its values tend to a value typical for the end of the first drive cycle.

This fact can be explained by energy accumulations in both the nominator and the denominator of the expression (7). The variation of $k_{E}$ for the multi-purpose vehicle is presented in Fig. 6 for the first (Fig. 6a) and the fifteenth (Fig. 6b) NEUDC cycle of the sequence. The higher values of $k_{E}$ at the beginning of the first cycle correspond to the values of the recuperation coefficient $k_{r}$ (relation (4)) because of the short periods of movements with constant speed. During the first $800 \mathrm{~s}$ of the NEUDC cycle the coefficient $k_{E}$ tends to a value determined by the small drive cycle ECE-15 (Fig. 6a). The alteration of the motion behaviour during the last $400 \mathrm{~s}$ of the cycle, changes the variations in the coefficient of $k_{E}$. This tendency in $k_{E}$ behaviour to come to a predetermined value, as a result of cyclic motion recurrence, is illustrated in Fig. 6b. The presented results allow the analysis for the achievable mileage and necessary initial energy levels of the vehicle energy sources to be carried out just over one cycle independently of the number of the reiterated cycles for the given mileage implementation.

\section{Energy recovery during brake modes and its impact on the mileage of a vehicle with conventional electric propulsion system}

It is considered a simple structural scheme of conventional electric propulsion system, as is shown in Fig. 7. The system contains an electric battery, an inverter, an induction electric motor with variable frequency drive control (VFD), and mechanical drive line of a clutch and a reduction gear. If the recuperation during braking modes is not taken into account, the mileage of such a vehicle can be estimated by energy balance in the following form:

$$
L=L_{C} \frac{Q_{b a t} \eta_{1}}{\Sigma E^{C}}
$$

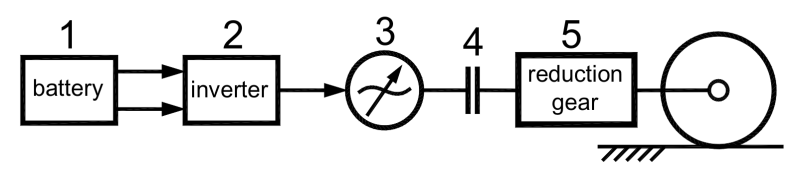

Fig. 7. A structural scheme of an electric vehicle contains an electric battery 1, an inverter 2, an AC electric motor with frequency drive control (VFD) control 3, a clutch 4 , reduction gear 5 and drive wheels 
where: $Q_{b a t}[\mathrm{kWh}]$ is available energy capacity of the battery, $\Sigma E^{C}=\Sigma E_{v i>v u i}$ $[\mathrm{kWh}]$ is the necessary energy to cover one cycle, but $L_{C}$ is the mileage of one cycle.

Taking into account the possibilities for energy recovery during brake modes and relation (7) for energy recovery coefficient $k_{E}$, the energy $\Sigma E^{C}$ needed to fulfil one cycle can be expressed by:

$$
\Sigma E^{C}=\Sigma E_{v i>v u i}-\Sigma E_{v i<v u i}=\left(1-\eta_{\Sigma} k_{E}\right) \Sigma E_{v i>v u i},
$$

and after replacing the expression above into the relation (9), the possible mileage is achieved as:

$$
L^{*}=L_{C} \frac{Q_{b a t} \eta_{1}}{\Sigma E^{C}}=L_{C} \frac{Q_{b a t} \eta_{1}}{\left(1-\eta_{\Sigma} k_{E}\right) \Sigma E_{v i>v u i}}=\frac{1}{\left(1-\eta_{\Sigma} k_{E}\right)} L,
$$

where: the term $\left(1-\eta_{\Sigma} k_{E}\right)^{-1}$ represents the possibilities for mileage increase of about $12-17 \%$ for overall efficiency of the drive line of $\eta_{\Sigma}=0.466$ and the different types of vehicles, presented in Table 1, at the same initial energy level of the electric battery (Fig. 8). Data from modelling of the electric vehicle i-Mi EV behaviour over the NEUDC cycle with energy recovery coefficient of $k_{E}=0.26$, are also added. The available data for the energy efficiency, concerning the electric vehicle i-Mi EV are obtained by using Japan's drive cycle JP 10-15 [13]. This cycle is an analogy of the European NEUDC cycle. A direct comparison between the calculated data according to relation (10) and

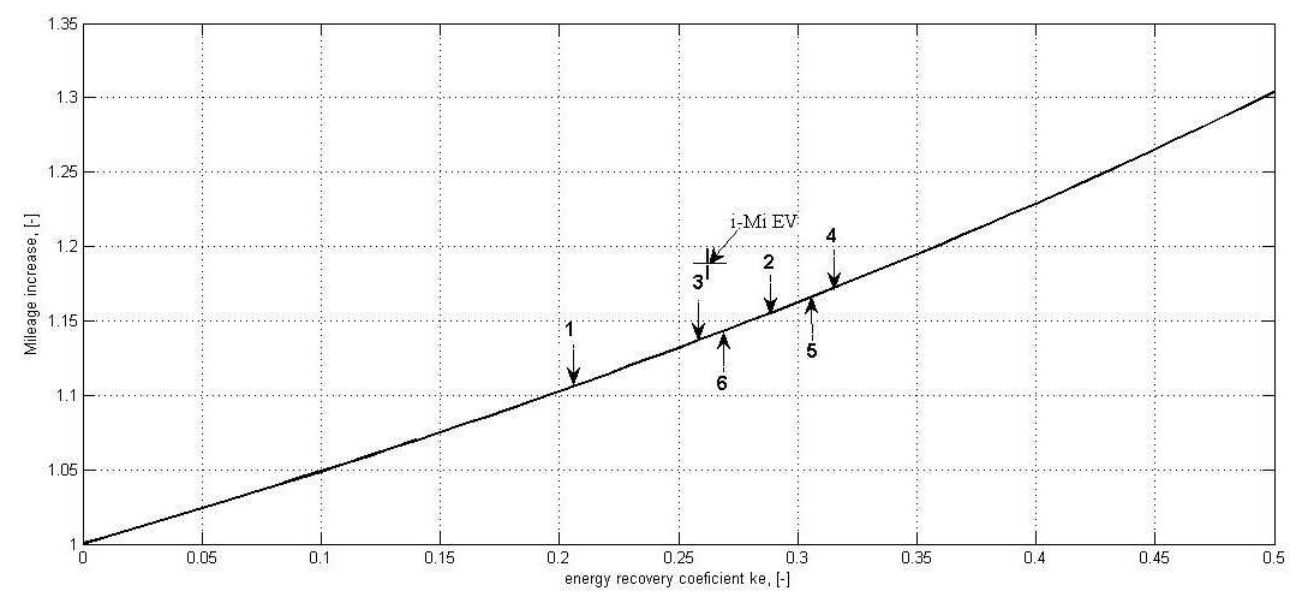

Fig. 8. Mileage increase over reiterated NEUDC cycles for the different vehicles, presented in Table 1 and an overall efficiency of the propulsion system of $\eta_{\Sigma}=0.466$ 
experimental ones is performed after solving the identification problem about rolling resistance coefficient and estimation of the overall efficiency of the i-Mi EV drive line over JP 10-15 cycle. The better result for i-Mi EV is due to the better efficiency of almost $\eta_{\Sigma}=0.60$ for its drive line.

\section{Structural variants of the hybrid electric propulsion systems with kinetic energy storage (KES)}

Advisability of energy utilization during brake modes is established based on the analysis of the energy flows and the numerical examples from the previous chapter. A flywheel energy source (KES) is considered as an energy storage. In case of electric vehicles, designed especially for urban traffic, KES usage as energy storage is suitable mainly in two structural modifications of the hybrid electrical drive line, as presented in Figs 9 and 10.

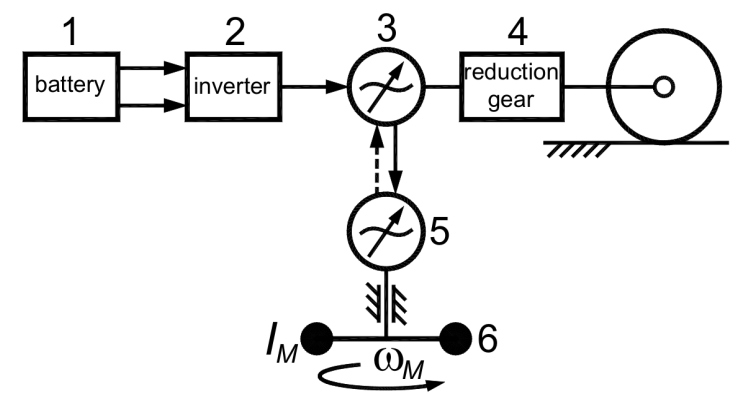

Fig. 9. A structural modification of a hybrid electrical drive line contained electric battery 1, inverter2, AC electric motors with VFD control 3 and 5, reduction gear 4 and KES 6

The first simplified scheme of the hybrid propulsion system for an electric vehicle is shown in Fig. 9. The components 1, 2, 3 and 4 set up the conventional electric propulsion system which allows energy recuperation during vehicle brake modes. The repeated sequences of accelerations and decelerations with high density of the power flows, especially in urban traffic, lead to shortening of the electric battery life. By building in an alternative energy source as KES (pos. 6), it is possible to smooth the pick loads over the battery during its charging/discharging modes. The KES source has different advantages, compared to the electric battery such as increased numbers of charge/discharge cycles or power transfers with higher intensity for examples. The KES coupling to the conventional propulsion system is realized electrically by means of an electric motor pos. 5 in Fig. 9. Such kind of connection allows high degree of 
flexibility during drive line design.

The considered hybrid drive line allows the following working modes: A. the entire recuperation energy during braking is directed to KES 6 at a maximum power output of the electric machine 5 ; $\mathrm{B}$. the electric battery is a main energy supply for all remaining driving modes if KES energy level is bellow a predetermined minimum one; C. if the energy in KES is over the predetermined minimum value, KES supports the electric battery with maximum power output of the electric machine 5 . This hybrid structure requires many transformations of the energy, which means more losses.

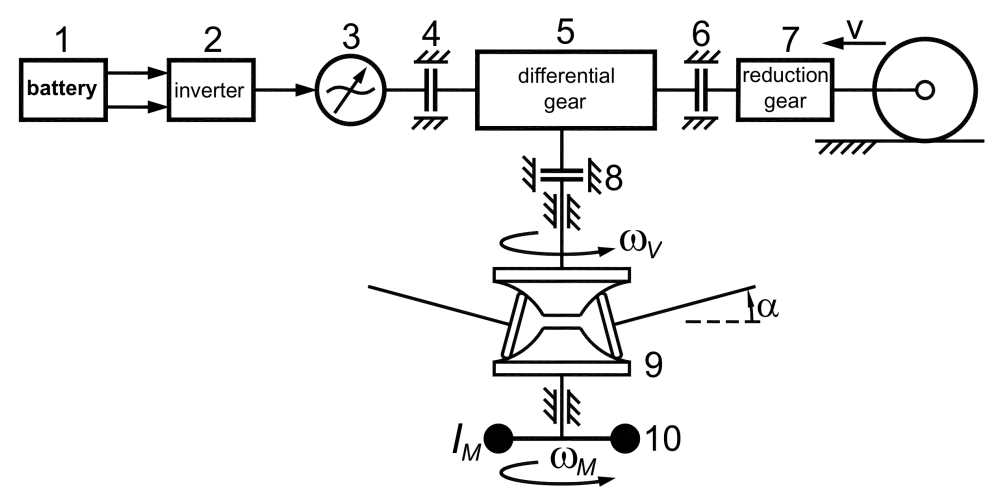

Fig. 10. A structural modification of a hybrid electrical drive line, consisted of an electric battery 1, an inverter 2, an AC electric motor with VFD control 3, clutchbrakes 4,6 , and 8 a differential gear 5, a reduction gear 7 friction variator 9 and

KES 10

The main advantage of the scheme in Fig. 10 is that, using an appropriate speed control by the control lever of the variator $\alpha=\alpha\left(\omega_{M}\right)$, the output variator speed $\omega_{V}$ can be kept constant $\omega_{V}=$ const [14], [15]. In such a condition, it is very easy to control the drive line output speed, i.e. the vehicle speed $v$ will be determined just only by the output speed of the AC electric motor 3 .

\section{Energy recovery coefficient $k_{E}$ in case of the hybrid electric propulsion system with KES}

The coefficient $k_{E}$, which is a drive cycle characteristic, can be expressed by the battery and KES energy levels alteration in the following form:

$$
k_{E}=\frac{\Delta E_{F E S}^{c h} / \eta_{4}+\left(\Delta E_{B a t}^{c h} / \eta_{2}\right) k_{1}}{\eta_{1} \Delta E_{\text {Bat }}^{\text {disch }}+\left(\eta_{3} \Delta E_{F E S}^{\text {disch }}\right) k_{2}},
$$


where: $\eta_{1}$ and $\eta_{3}$ are the overall efficiency coefficients of the drive lines from the battery and KES, respectively; $\eta_{2}$ and $\eta_{4}$ are the overall efficiency coefficients of the drive lines from the driven wheels to the battery and KES, respectively; $\Delta E^{\text {ch/disch }}$ are the energy alterations of the two alternative energy storage devices - the battery and KES; coefficients $k_{1}$ and $k_{2}$ represent the limiting conditions concerning the KES modes of operation. Using the relation $S o C=$ $E_{B a t} / E_{B a t 0}$, which represents the charge state of the electric battery and a similar expression for KES, the cycle coefficient $k_{E}$ can be presented in the following form:

$$
k_{E}=\frac{\Delta S o C_{F E S}^{c h} / \eta_{4}+\left(\Delta S o C_{B a t}^{c h} / \eta_{2}\right) k_{B a t / F E S} k_{1}}{\eta_{1} \Delta S o C_{B a t}^{\text {disch }} k_{\text {Bat } / F E S}+\left(\eta_{3} \Delta S o C_{F E S}^{\text {disch }}\right) k_{2}},
$$

where: the different coefficients $k_{1}, k_{2}$ and $k_{B a t / F E S}$ are determined as:

$$
\begin{aligned}
& k_{\text {Bat } / F E S}=\frac{E_{\text {Bat }}^{\max }}{E_{F E S}^{\max }} ; \\
& k_{1}=\left\{\begin{array}{c}
0, S o C_{F E S} \neq 1 \\
1, S o C_{F E S}=1
\end{array} ; \quad k_{2}=\left\{\begin{array}{c}
0, \text { SoC } C_{F E S} \leq 0.1 \\
1,0.1 \leq S o C_{F E S} \leq 1
\end{array},\right.\right.
\end{aligned}
$$

at $S o C_{B a t}=S o C_{B a t 0}-\Delta S o C_{B a t}^{\text {disch }}+\Delta S o C_{B a t}^{c h}$ and $S o C_{F E S}=S o C_{F E S 0}-\Delta S o C_{F E S}^{\text {disch }}+\triangle S o C_{K A E}^{c h}$.

The coefficient $k_{1}$ is determined by the range, where KES is able to store the energy during braking modes. In cases, when the KES is unable to store any additional energy (KES is fully charged, for example), the energy available for recuperation is transferred to the electric battery and the coefficient switches to a value of $k_{1}=1$. The coefficient $k_{2}$ corresponds to mode $\mathrm{B}$ (described in chapter 3), when it is suitable to use the energy stored into KES to support the electric battery to cover the vehicle energy demands.

\section{Initial energy levels for the alternative energy sources}

The following analysis and numerical results are based on modelling of multi-purpose vehicle (type 5 from Table 1). It is assumed that this vehicle possesses the electric hybrid propulsion system, presented in Fig. 9, but with the following specific characteristics: the prime electric motor 3 with nominal power of $65 \mathrm{~kW}$, and the $25 \mathrm{~kW}$ power secondary electric motor 5 . The accepted power values are chosen by taking into consideration the specific power 
demands for fulfilling the already mentioned drive cycles. The following additional data are used during the numerical modelling process: efficiency of the energy transfer paths: $\eta_{M 3}=\eta_{M 5}=0.9$ are the mechanical efficiency coefficients of the electric motors 3 and 5 , which are independent of the direction of the energy transfer; $\eta_{B a t}=0.85 / 0.8$ is the electric battery internal efficiency during charging, discharging processes, respectively; $\eta_{F D}=0.92$ is the overall mechanical efficiency of the reduction gear 4 and the final drive.

Initial energy level of the primary source (electric battery)

In case of conventional electrical propulsion system, the coefficient of energy recovery $k_{E}$ (relations 7 and 10) can be expressed by the energy stored in the electric battery:

$$
k_{E}=\frac{\Delta E_{\text {Bat }}^{c h} / \eta_{2}}{\eta_{1} \Delta E_{\text {Bat }}^{\text {disch }}}=\frac{\Delta S o C_{B a t}^{c h} / \eta_{2}}{\eta_{1} \Delta S o C_{B a t}^{\text {disch }}},
$$

where: $\Delta E_{B a t}^{\text {disch }}$ and $\Delta E_{B a t}^{c h}$ are the energy changes of the battery state, which directly correspond to the cycle energy demands $\Sigma E_{v i>v u i}$ and $\Sigma E_{v i<v u i}$, but $\eta_{1}$ and $\eta_{2}$ represent the overall efficiencies of the propulsion system in the both main battery modes - discharging and charging, respectively. The battery parameter state of charge $S o C=E_{B a t} / E_{B a t 0}$ can be expressed by the first relation of (11), where $S o C_{B a t 0}$ describes the initial battery energy (usually $\left.S o C_{B a t 0}=1\right)$.

From the relation (13) and the first relation from (11), for known values of $k_{E}$, i.e. chosen drive cycle, and overall efficiencies $\eta_{1}$ and $\eta_{2}$, it follows that the current battery state of charge is:

$$
S o C_{B a t}=S o C_{B a t 0}-\left(\frac{1}{\eta_{1}}-\eta_{2} k_{E}\right) \frac{\Sigma E_{v i>v u i}}{E_{B a t}^{\max }} .
$$

If a preliminary condition is attached for full battery discharge at the end of the proposed vehicle mileage $\left(S o C_{B a t}=0\right)$, the minimum battery energy state necessary for implementation of the same mileage is calculated as:

$$
E_{B a t}^{\max }=\left(\frac{1}{\eta_{1}}-\eta_{2} k_{E}\right) \frac{\Sigma E_{v i>v u i}}{S o C_{B a t 0}} \frac{L_{d}}{L_{C}} .
$$

The covered distance is $L_{C}=10.84 \mathrm{~km}$ on one NUEDC cycle. The condition of desired mileage of $L_{d}=160 \mathrm{~km}$ only using NEUDC cycle, at $\eta_{2}=0.6624$ and $k_{E}=0.3073$ leads to the following results: 
On the Modulation of Oscilation in Thermohaline Convection Problems ... 31

- Conventional electric propulsion system without recuperation - using relation (8), which can be solved against the battery capacity of $Q_{b a t}$, or using relation (13) at $k_{E}=0.0$ : both variants lead to $Q_{b a t} \equiv E_{B a t}^{\max }=31.95$ kWh.

- Conventional electric propulsion system with recuperation - using relation (9), which can be solved against the battery capacity of $Q_{b a t}$, or using relation (13): both variants lead to $Q_{b a t} \equiv E_{B a t}^{\max }=27.37 \mathrm{kWh}$.

The latter result, concerning the case with recuperation, can be transformed into additional vehicle mileage. This results in $15.9 \%$ increase in the achievable mileage over NEUDC cycle, the fact which coincides completely with the results from chapter 2 .

Initial energy level of the secondary source (KES)

KES maximum energy level determination is a complex task. The higher available energy levels allow the higher density of the power density over a time, but also mean mass or velocity increase. The main criterion here would be the condition for cyclic recurrence of the KES usage, i.e. what is the optimum for KES energy level for given vehicle and drive cycle, which ensures the KES states of charge at the beginning and at the end of the one drive cycle to be almost equal. The condition above can be fulfilled independently by active reduction of the energy flow from KES, which will be discussed further in the next chapter. For this purpose, a power reduction coefficient for power is introduced. Meanwhile, in case of lack of enough initial data for the hybrid drive line, the most appropriate criterion for KES energy level calculation is the all available energy for recuperation from one cycle to be stored into KES. brake modes

KES initial energy level according to a full energy recovery condition at

The idea that all dissipated energy during braking over one cycle has to be stored into KES is reasonable, as the KES maximum level is very easy to calculate without taking into account KES's discharge processes. In such a condition, the coefficient $k_{1}$ in the relation (11) has to be zeroed. By comparing the numerators in relations (4) and (13), where the relation (13) is rewritten for the KES, it is obtained the following relation:

$$
\left|\Sigma E_{v i<v u i}\right|=\frac{1}{\eta_{4}} \Delta E_{F E S}^{c h}=\frac{1}{\eta_{4}} \Delta S o C_{F E S}^{c h} E_{F E S}^{\max },
$$

which solution, according to the maximum energy level for KES, is:

$$
E_{K A E}^{\max }=\frac{\eta_{4}\left|\Sigma E_{v i<v u i}\right|}{\Delta S o C_{F E S}^{c h} \max } .
$$


It is accepted in the theory, developed for KES, that the permissible maximum change of the KES state of charge is $\triangle S o C_{F E S}^{c h} \max =0.9\left(\frac{w_{F E S}^{\max }}{w_{F E S}^{\min }} \approx 3\right.$ at fixed mass parameters), and using the same initial data for the vehicle and the drive cycle, i. e. $\left|\Sigma E_{v i<v u i}\right|=0.468 \mathrm{kWh}$ and $\eta_{4}=0.7452$; the required energy level of KES is $E_{K A E}^{\max }=0.3875 \mathrm{kWh}$.

\section{KES possibilities for mileage increasing of vehicles with elec- tric propulsion system}

Coefficient of power reduction

Optimum usage of KES as an alternative energy source requires acceptable KES state of charge over the entire drive cycle. This requirement allows at any energy demand over the drive cycle and chosen strategy of KES usage, the KES energy levels to be sufficient to cover such a demand [16]. The energy recovery coefficient $k_{E}$ in case of hybrid electric propulsion system, relation (11), is accepted as a starting point for initial KES state of charge estimation. The KES state of charge has two upper and lower limits. KES is unable to store the available energy during brake mode at its upper limit, which corresponds to fully charged KES at $S O C_{F E S}=1$. On the contrary, if $S o C_{F E S}=0.1$, which corresponds to the minimum acceptable discharged level of KES, it is impossible to use KES as an alternative source during vehicle acceleration modes. In both cases, the KES usage as energy buffer storage is compromised, which leads to the first constraint condition in relation (11) $-k_{2}=1$. While keeping the electric battery as safe as possible, it is also desirable that all available energy for recuperation during brake modes should be stored into KES, i. e. the second coefficient in relation (11) has to be zeroed $\left(k_{1}=0\right)$. Thus, the relation (11) for the energy recovery coefficient $k_{E}$ is transformed as:

$$
k_{E}=\frac{\Delta S o C_{F E S}^{c h} / \eta_{4}}{\eta_{1} \Delta S o C_{B a t}^{\text {disch }} k_{B a t / F E S}+\left(\eta_{3} \Delta S o C_{F E S}^{\text {disch }}\right)} .
$$

The solution of the above relation according to the electric battery state of discharge $\Delta S o C_{B a t}^{\text {disch }}$ is:

$$
\Delta S o C_{B a t}^{\text {disch }}=\frac{1}{\eta_{1} k_{B a t / F E S}}\left(\frac{\Delta S o C_{F E S}^{c h}}{\eta_{3} k_{E}}-\eta_{3} \Delta S o C_{F E S}^{\text {disch }}\right),
$$

If it is considered that the denominator is a constant i. e. $\eta_{1} k_{B a t / F E S}=$ const, the battery state of discharge has an extremum at:

$$
\frac{\Delta S o C_{F E S}^{c h}{ }^{\prime}}{\eta_{4} k_{E}}-\eta_{3} \Delta S o C_{F E S}^{d i s c h h^{\prime}}=0
$$


which leads to:

$$
\Delta_{F E S}^{\text {disch' }}=\frac{\Delta S o C_{K A E}^{c h}{ }^{\prime}}{\eta_{3} \eta_{4} k_{E}}=k_{P} \Delta S o C_{F E S}^{c h}{ }^{\prime}
$$

The variables $\triangle S o C_{F E S}^{c h}{ }^{\prime}$ and $\Delta S o C_{F E S}^{\text {disch }^{\prime}}$ are equivalent with accuracy to a constant, depending on time to the intensity of the energy change of the KES state $-\triangle E_{F E S}^{c h}$ ' and $\Delta E_{F E S}^{\text {disch }^{\prime}}$, respectively. By presenting the variables $\triangle E_{F E S}^{c h}{ }^{\prime}$ and $\Delta E_{F E S}^{\text {disch' }}$ in discrete form, which correspond to the drive cycle representation, it is possible to make an analogy to the power transfer to and from the KES, i. e. $\Delta E_{F E S i}^{c h} \rightarrow P_{F E S i}^{c h}$ and $\Delta E_{F E S i}^{\text {disch }}{ }^{\prime} \rightarrow P_{F E S i}^{\text {disch }}$, and after substitution into the relation (15), it is received:

$$
P_{F E S i}^{\text {disch }}=k_{P i} P_{F E S i}^{c h},
$$

where: $k_{P i}=\left(\eta_{3} \eta_{4} k_{E i}\right)^{-1}$ are transitory values for power reduction coefficient for the electric machine (pos. 5 in Fig. 9), coupled to KES. After prolonged movement over the same drive cycle, the values of $k_{E i}$ tend to the specific value for $k_{E}$, which is representative for the given drive cycle (see Table 1 and Fig. 6), whence the power reduction coefficient tends to:

$$
k_{P}=\left(\eta_{3} \eta_{4} k_{E}\right)^{-1},
$$

which is again a typical value for given vehicle and drive cycle.

The relation (20) shows that following the idea for adequate energy levels for KES usage during the defined drive cycle, the power flow from KES would be considered as a functional dependence on both the speed recovery of the energy (power transfer for charging), stored in KES, and vehicle driving modes taking into account the energy losses.

Power reduction coefficient impact on energy states of both energy sources and achievable mileage at fixed initial energy levels of the sources

The described power reduction coefficient concerns the maximum power of the electric motor pos. 5 coupled to KES (Fig. 9). The entire capacity of this electric machine is used at $k_{P}=1$, when it is in an operation generator mode. The increase of the coefficient $k_{P}$ (which corresponds to $k_{E}$ decrease) means a decrease of the maximum power, which can be used by KES taking into account possibilities for energy recuperation. In this way, the KES energy state can be controlled over the drive cycle on the expense of the energy level of the electric battery. This control assumes better utilization of KES energy, as this does not allow prolonged periods of usage of KES at its boundary states (maximum 
charged, maximum discharged state respectively), which leads to a necessity to switch off the KES branch from the propulsion system. The results from numerical modelling of the energy transfers into the hybrid propulsion system of an multi-purpose vehicle over NEUDC are shown in Fig. 11. The energy levels of the alternative energy sources (electric battery and KES) are presented by their states of charges in the following basic modes of operation: all the energy demands are covered entirely by the electric battery - the conventional electric propulsion system without recuperation; all the energy demands are covered by the electric battery - the conventional electric propulsion system with recuperation; the energy demands are covered by the both energy sources -electric battery and KES, observing the described possible modes of the hybrid system. It is accepted an initial energy level of $4 \mathrm{kWh}$ for the electric battery, which allows mileage over two NEUDC. It is also accepted an initial KES energy capacity of $0.3848 \mathrm{kWh}$, determined by the condition for full energy recuperation - relation (15). Those initial values give a clearness of the results over just one drive cycle. The energy consumption in the three variants shown in Fig 11 is better, than the one in case of conventional electric propulsion with recuperation, due to the better efficiency of the power branch to and from KES. The fixed power reduction from KES seems to be the optimal variant for KES usage in case of cyclic recurrence in the vehicle motion.

The influence of the power reduction coefficient is illustrated by maximum achievable mileage of the mentioned vehicle until the electric battery becomes fully exhausted. The vehicle motion is modelled over repeated NEUDC cycle at fixed initial energy states for the electric battery and KES: fully charged battery and KES with capacity of $31.95 \mathrm{kWh}$, and $0.3848 \mathrm{kWh}$, respectively. The results from the modelling are presented in Table 2 and they describe the achievable mileage as a function of the power reduction coefficient $k$ which is reciprocal to $k_{P}$ (relation $(20)$ ).

Two typical cases of the initial available energy states for KES - fully charged, and fully discharged, respectively are considered. In both cases, the maximum of the achievable mileage is at $k_{P} \approx 5$, which corresponds to the values from relation (20). Numerical modelling is performed, using the following data: a typical multi-purpose vehicle according to Table 1, and maximum power of $25 \mathrm{~kW}$ for the electric machine coupled to the KES. The main difference here is the fact, that only one fifth of this power is used during acceleration and additional power for covering the energy demands, which is supplied by the electric battery. The comparative analysis with the conventional propulsion system shows an increase of $22.7 \%$ in achievable mileage in case of initially fully charged KES. 


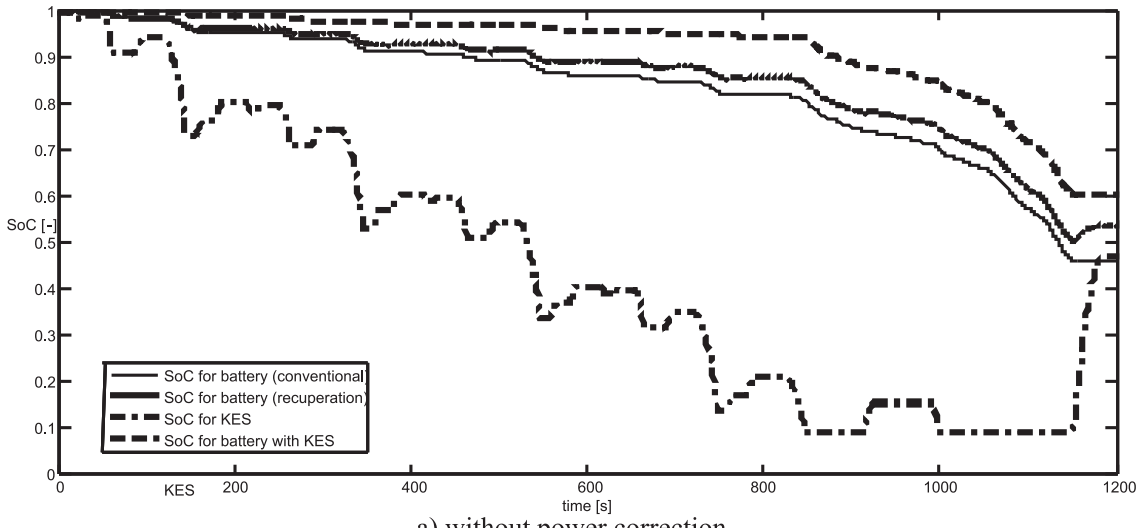

a) without power correction

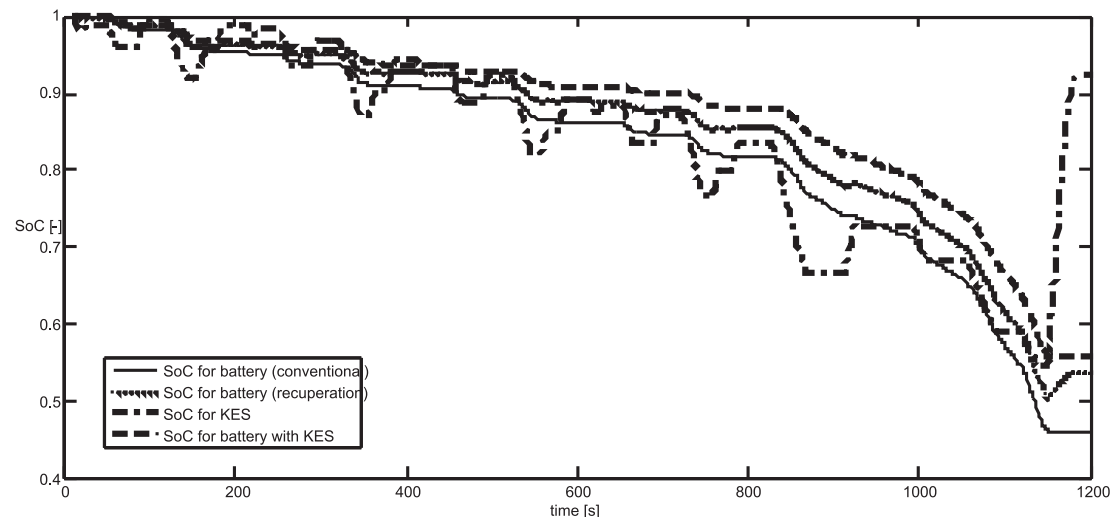

b) with adaptive power correction

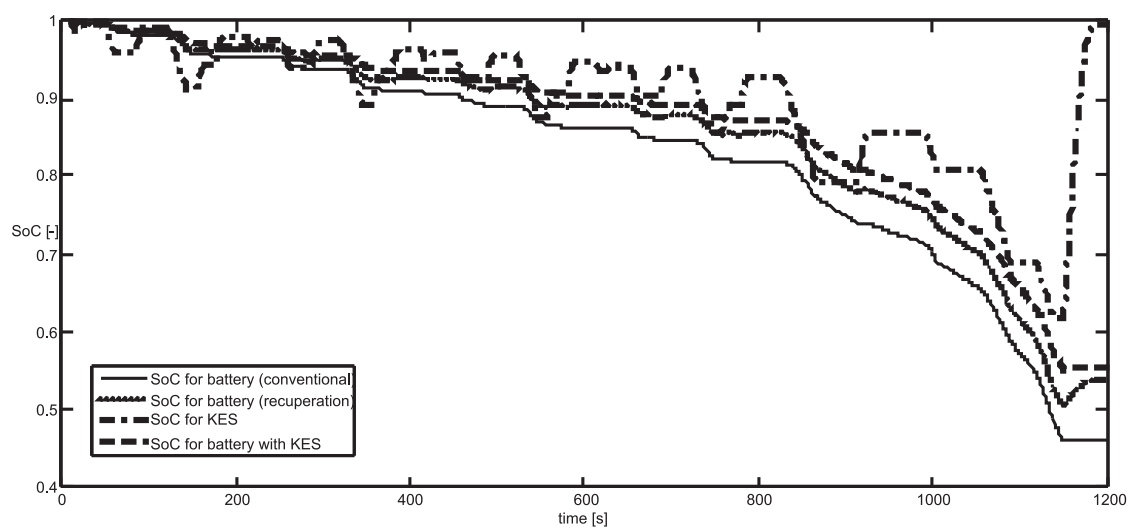

c) with fixed power correction, which ensures full energy recovery of KES at the end of the cycle

Fig. 11. Energy states of the alternative sources over NEUDC drive cycle 
Table 2. Influence of the power reduction coefficient on the achievable mileage over NEUDC drive cycle

\begin{tabular}{|c|c|c|c|c|}
\hline \multicolumn{5}{|c|}{ NEUDC } \\
\hline \multicolumn{2}{|c|}{ Full KES } & \multicolumn{2}{|c|}{ Empty KES } & SOC of KES \\
\hline $\mathrm{k}$ & $\mathrm{S}[\mathrm{km}]$ & $\mathrm{k}$ & $\mathrm{S}[\mathrm{km}]$ & to bottom limit \\
\hline 1 & 191.05 & 1 & 186.75 & $\Uparrow$ \\
\hline 2 & 192.63 & 2 & 188.54 & \\
\hline 3 & 193.56 & 3 & 189.81 & \\
\hline 4 & 193.71 & 4 & 190.29 & \\
\hline 5 & 193.83 & 5 & 190.32 & \\
\hline 6 & 190.30 & 6 & 186.70 & $\Downarrow$ \\
\hline 7 & 187.95 & 7 & 183.67 & to upper limit \\
\hline
\end{tabular}

At low values for $k$, i.e. the maximum usage of the electric machine 5, the average KES state of charge, which is kept by recuperation, is not enough to cover all the drive cycle energy demands, thus the electric battery has to be used to compensate the shortage of power, but with its lower efficiency. On the contrary, at high values for $k$ the power reduction of the flow from KES has a negative effect by keeping the KES state of charge at its upper limits, which leeds to the loss of possibility to store recuperation energy into KES. The extremum of the mileage is reached at value which corresponds to the drive cycle energy recovery coefficient, according relation (20). By comparing the mileage in the both considered cases: fully charged and fully discharged $\mathrm{KES}$, it becomes clear that the available energy in KES, when the KES is fully charged, is enough for covering a mileage of 3.5 to $4 \mathrm{~km}$. This confirms the idea that KES must be used as a buffer accumulator instead of a main one.

The data presented in Table 3 summarize the KES influence over the entire efficiency of the described multi-purpose vehicle with electric propulsion system over NEUDC cycle. The results are expressed by achievable mileage in case of fixed optimum value for power reduction coefficient $k_{P}$ of 5.1 (Table 2 ). The results coinside with the calculations made in section 3: an increase of $15.1 \%$ of the achiavable mileage due to recuperation in case of a conventional propulsion system; an increase of $22.7 \% / 20.6 \%$ in the achievable mileage in case of the hybrid propulsion system with battery and initially fully charged KES, fully discharged KES, respectively.

\section{Conclusion}

Based on the theoretical investigations, a real possibility is determined for $25-40 \%$ energy recovery during brake modes in city urban drive cycles. An 
On the Modulation of Oscilation in Thermohaline Convection Problems ... 37

Table 3. General results of motion over NEUDC cycle

\begin{tabular}{|l|c|c|c|}
\hline NEUDC & $\begin{array}{c}\text { Mileage, } \\
\mathrm{km}\end{array}$ & $\begin{array}{c}\text { Duration, } \\
\mathrm{h}\end{array}$ & $\begin{array}{c}\text { Number of } \\
\text { cycles }\end{array}$ \\
\hline $\begin{array}{l}\text { Electric battery supply without recu- } \\
\text { peration }\end{array}$ & 157.75 & 4.95 & 15 \\
\hline $\begin{array}{l}\text { Electric battery supply with recupera- } \\
\text { tion }\end{array}$ & 181.6 & 5.65 & 17 \\
\hline $\begin{array}{l}\text { Hybrid supply with fully charged/dis- } \\
\text { charged KES }\end{array}$ & $193.8 / 190.3$ & $6.0 / 5.95$ & 18 \\
\hline
\end{tabular}

application of a hybrid drive line for electric propulsion system with kinetic energy storage, as an alternative energy source is investigated. KES usage as an energy buffer in the electrical propulsion system has twofold impact on such vehicles: better electric battery modes of operation and increased mileage. Taking into account the internal energy losses, the results show an increase between $15 \%$ and $23 \%$ of the achievable mileage of a vehicle with mass of 1700 $\mathrm{kg}$ (multi-purpose vehicle) over NEUDC cyclic recurrence until the main energy source - the electric battery becomes fully discharged.

Mileage improvement is derived by transferring a part of energy flows through KES more efficient branch of the propulsion system. It is shown, that further mileage increase is possible, if the power flows during KES depletion are considered as a function of both the concrete driving cycle and corresponding modes, and the speed of KES energy recovery during braking modes. Further investigations will take into account driver behaviour and how different behaviours influence on energy levels, and achievable mileage.

\section{REFERENCES}

[1] Ehsani, M., et al. Modern Electric, Hybrid Electric, and Fuel Cell Vehicles, CRC press, 2005.

[2] Larminie, J., J. Lowry. Electric Vehicle Technology Explained, John Wiley \& Sons, 2003.

[3] Pandey, A., A. Jain, V. Arora, S. Sharma. Integration and Performance Analysis of Flywheel Energy Storage System in a ELPH Vehicle, Int. Joint Journal Conference in Engineering, IJJCE'2009, 1 (2009), No. 5.

[4] Jivkov, V. The Flywheel of the 21th Century, 11 ${ }^{\text {th }}$ International Scientific Conference of the Association of Machine Technology Faculties in Bulgaria AMTECH'2012, 19-20 Oct., Bulgaria, Sofia, 2-12, 27-41 (in Bulgarian). 
[5] Fu, X., H. Wang, N. Cui, C. Zhang. Energy Management Strategy Based on the Driving Cycle Model for Plug in Hybrid Electric Vehicles, Abstract and Applied Analysis, vol. 2014, article ID 341096, 2014.

[6] http://www.dieselnet.com/standarts/cycles

[7] Gonder, J., T. Markel. Energy Management Strategies for Plug-in Hybrid Electric Vehicles, SAE 2007-01-0290, 2007.

[8] Lin, C., J. Kang, J. Grizzle, H. Peng. Energy Management Strategy for a Parallel Hybrid Electric Truck, IEEE Transactions on Control Systems Technology, 11 (2003), No. 6, 839-849.

[9] Moura, S., D. Callaway, H. Fathy, J. Stein. Impact of Battery Sizing on Stochastic Optimal Power Management in Plug-in Hybrid Electric Vehicles, ICVES 2008, IEEE International Conference, 2008, 96-102.

[10] Zou, Y., H. Shi-Jie, L. Dong-Ge, G. Wei, X. Hu. Optimal Energy Control Strategy Design for a Hybrid Electric Vehicle, Discrete Dynamics in Nature and Society, vol. 2013, 2013, article ID 132064.

[11] Pandey, A., H. Bansal. A Review of Optimal Energy Management Strategies for Hybrid Electric Vehicle. International Journal of Vehicular Technology, 2014 (2014), article ID 160510.

[12] Guzzella, L., A. Sciarretta. Vehicle Propulsion Systems, 2-nd Edition, Springer Berlin, Heidelberg New York, 2007.

[13] Kozinori, H., H. Yoshida. Development of Next-Generation Electric Vehicle Mitsubishi - Mi EV. ATZ Auto Technology, 8 (2008), No. 10, 18-23.

[14] Jivkov, V., K. Stoichkov. Kinetic Energy Storage Control at Idle Mode by Friction Variator. Machine Mechanics, 85 (2008), 47-52 (in Bulgarian).

[15] Stoichkov, K., V. Jivkov, N. Nikolov. Analysis and Synthesis of a Friction Mechanism with Minimum Sliding, Sofia, Bultrib-2009, 54-58 (in Bulgarian).

[16] Rahman, Z., K. Butler, M. Ehsani. A Study of Design Issues on Electrically Peaking Hybrid Electric Vehicle for Diverse Urban Driving Patterns, SAE 199901-1151, 1999. 